\title{
Actitudes hacia la homosexualidad y personas trans en estudiantes de educación física de Chile \\ Attitudes towards homosexuality and trans people in physical education students in Chile
}

\author{
*Fernando Maureira Cid, **Natalia Escobar Ruiz,**Elizabeth Flores Ferro, *Valentina Bahamonde Acevedo, \\ ***Marcelo Hadweh Briceño, ****Daniel Serey Araneda \\ *Universidad Metropolitana de Ciencias de la Educación (Chile), **Universidad Bernardo O’Higgins (Chile), \\ ***Universidad SEK (Chile), ****Universidad Católica del Maule (Chile)
}

\begin{abstract}
Resumen. Introducción: La homofobia se entiende como el sentimiento de rechazo o temor hacia una persona homosexual. La discriminación y desaprobación hacia las personas denominadas como transgénero o transexual es conocido como transfobia. Ambas actitudes están muy presentes en la Educación Física, por eso el objetivo de la presente investigación fue validar dos escalas de actitudes negativas hacia la homosexualidad y personas trans en estudiantes de Educación Física de Chile y describir sus actitudes. Método: se aplicó la escala de homofobia EHF-10 y la escala de actitudes negativas hacia personas trans a 255 estudiantes de Pedagogía en Educación Física de tres universidades de Chile. Resultados: los dos instrumentos presentan niveles adecuados de validez y confiabilidad. Las afirmaciones: Me avergonzaría tener un hermano homosexual o hermana lesbiana y Las personas trans no deberían poder enseñar en las escuelas poseen el mayor porcentaje de respuestas totalmente en desacuerdo y en desacuerdo con más del 90\%. Los resultados de la escala de homofobia EHF-10 no presentan diferencias significativas entre hombres y mujeres y en la escala de actitudes negativas hacia personas trans sólo presenta diferencias en uno de sus ítems. Conclusión: ambas escalas son válidas y confiables para ser aplicadas a estudiantes de Educación Física. Se observan bajos niveles de homofobia y transfobia en la muestra.
\end{abstract}

Palabras claves: homofobia, transfobia, educación física, estudiantes.

\begin{abstract}
Introduction: Homophobia is understood as the feeling of rejection or fear towards a homosexual person. Discrimination and disapproval of people referred to as transgender or transgender is known as transphobia. Both attitudes are very present in Physical Education, so the objective of this research is to validate two scales of negative attitudes towards homosexuality and trans people in students of Physical Education of Chile and describe their attitudes. Method: the homophobia scale EHF-10 and the scale of negative attitudes towards trans people were applied to 255 students of Physical Education Pedagogy from three universities in Chile. Results: The two instruments have adequate levels of validity and reliability. The statements: I would be ashamed to have a gay brother or lesbian sister and Trans people should not be able to teach in schools have the highest percentage of responses totally disagreeing and disagreeing with more than 90\%. The results of the homophobia scale EHF10 present no significative differences between men and women and in the scale of negative attitudes towards trans people only present differences in one of its items. Conclusion: Both scales are valid and reliable to be applied to students of Physical Education. Low levels of homophobia and transphobia were observed in the sample.
\end{abstract}

Keywords: homophobia, transphobia, physical education, students.

\section{Introducción}

La orientación sexual corresponde a una dirección de sentimientos y conductas eróticas hacia alguien de sexo opuesto (heterosexual), hacia el mismo sexo (homosexual), ambos (bisexual) o ninguno (asexual), lo cual involucra la atracción afectiva, romántica y sensual (American Psychological Association, 2011). Por su par-

Fecha recepción: 19-04-21. Fecha de aceptación: 14-06-21

Fernando Maureira Cid

maureirafernando@yahoo.es te, la identidad de género corresponde a la autopercepción subjetiva que cada individuo tiene sobre su género (Maureira, 2020), es decir, una autoidentificación como hombre o mujer sobre la base de lo que culturalmente se entiende por hombre o mujer (García, 2005). Esta autopercepción puede coincidir con el sexo asignado al nacer (cisgénero) o puede ser diferente (transgénero). Autores como Páez et al. (2015) incluyen dentro de las personas trans a transgéneros, transexuales y travestis.

La homofobia se entiende como el sentimiento de rechazo, temor o repugnancia hacia una persona homosexual (Campo et al. 2013; Borrillo, 2010), lo que con- 
lleva a un comportamiento hostil que incluye maltrato verbal y violencia física (Silva et al. 2018). Sin embargo, Barrientos y Cárdenas (2013) indican que no es adecuado en la actualidad considerarla necesariamente una fobia, sino más bien, como una hostilidad, ya que en las personas homofóbicas provoca emociones de ira y enojo en vez de ansiedad. Por otra parte, la discriminación y rechazo hacia las personas denominadas como transgénero o transexual es conocido como transfobia (Barrientos et al. 2019), el cual, explica Carmona (2021) busca reconocer sólo el sexo biológico (mujer y hombre), en personas que nacieron biológicamente hombres, pero que se sienten y viven como mujeres o biológicamente son mujeres pero que sienten y viven como hombres (Carvajal, 2018).

En la escuela, la asignatura de Educación Física es el área que presenta más rechazo a la diversidad sexual, con altos niveles de homofobia y, por lo tanto, donde hay más trabajo que realizar (Ayvazo y Sutherland, 2009, McCaughtry, et al., 2005). Esta misma situación se presenta claramente en el deporte, con rechazo hacia homosexuales y lesbianas (Barbero, 2003, O'Brien, et al., 2013), con estereotipos negativos, acoso verbal, discriminación, aislamiento, etc. hacia dichos deportistas (Barber \& Krane, 2007). Para Ayvazo y Sutherland (2009) las visiones de masculinidad y feminidad están más acentuadas en el ámbito deportivo.

Un estudio de O'Brien et al. (2013) examinó los niveles y razones de la homofobia en estudiantes universitarios de Educación Física y estudiantes de otras carreras en Nueva Zelanda. Los primeros presentaron un mayor prejuicio anti-homosexual y anti-lesbiana, con un mayor prejuicio anti-homosexuales pero no antilesbianas en los varones. Estos prejuicios se asociaron a la agresión autoritaria y orientación al dominio social. Piedra et al. (2013) con estudiantes universitarios de Educación Física de España reveló que, durante su educación secundaria, un $46.9 \%$ fue testigo muchas veces y un $33.1 \%$ fue testigo algunas veces de comportamientos heterosexistas en las clases de Educación Física, y consideran que este profesorado está poco concienciado sobre el heterosexismo y la homofobia en las clases de Educación Física. Otro trabajo de Piedra et al. (2014) muestra que los/as profesores/as de Educación Física tienen un trato respetuoso y cordial con niños/as homosexuales, sin embargo, reproducen inconscientemente estereotipos con sus estudiantes, los que sufren discriminaciones y agresiones. Gill et al. (2010) describe que los estudiantes universitarios de Ciencias del Deporte perciben un ambiente hostil hacia homosexuales, con exclusión e insultos, en clases de Educación Física. Piedra et al. (2016) aplicaron un cuestionario de percepción de la homofobia y heterosexismo en clases de educación física a 170 profesores/as escolares de la especialidad, revelando evidentes comportamientos homofóbicos por parte de los estudiantes, con lenguaje discriminador a homosexuales y lesbianas. Silva et al. (2018) observaron comportamientos heterosexistas y homofóbicos en las clases de Educación Física, con mayor prevalencia de las creencias de una superioridad masculina, donde las mujeres tienden a ser más perceptivas de esta situación. Beserra et al. (2019), en un estudio con 40 estudiantes, 3 profesores y 1 profeso$\mathrm{ra}$, revelaron agresiones verbales a homosexuales durante la clase de Educación Física.

Márquez (2017) describe que el 88.8\% de una muestra de estudiantes universitarios de diferentes asignaturas, incluida Educación Física, expresan que se debiese respetar la homosexualidad independientemente del contexto, lo que no concuerda con las entrevistas que revelan exclusión social ante la homosexualidad al ser algo anormal, lo que en Educación Física se agudiza por presentar actitudes machistas y sexistas generando actitudes de discriminación. Serra et al. (2020) analizaron el currículum asociado a la perspectiva de género en la formación de profesores de Educación Física de 81 instituciones de educación superior de Brasil y España encontrando principalmente que la perspectiva de género de las instituciones está desarticulada de los marcos legislativos y que se debiese incorporar por compromiso social y no sólo para cumplir la legislación vigente. En el trabajo de Guevara (2020) los hallazgos indican que los estudiantes universitarios de Educación Física heterosexuales masculinos presentan mayor nivel de rechazo a la homosexualidad masculina que estudiantes femeninas y de ambos sexos. Lleixá et al. (2020) realizó una investigación acerca de la perspectiva de género en la formación docente de Educación Física, en la cual, se concluye que es vital transformar los modelos y relaciones tradicionales de género, por lo tanto, es necesario que exista reflexión crítica y las herramientas para abordar el cambio en las instituciones educativas responsables. Situación que concuerda con la investigación de Camacho-Miñano y Girela-Rejón (2017) acerca de la evaluación de una propuesta formativa acerca de género en Educación Física, donde se concluye que siendo una experiencia formativa no obligatoria ni estar dentro del plan de estudio, al no tener continuidad pierde eficacia y sentido para lograr cambios trascendentales en los estudiantes. 
Un estudio realizado con estudiantes de Educación Física develó que su imaginario sobre personas trans lo relacionan con la compasión, genitalización, violencia simbólica y estigmatización (Pérez et al., 2016). Personas transexuales describen las clases de Educación Física en su etapa escolar como una experiencia de constante soledad y aislamiento, padecimiento de situaciones de estigmatización y acoso, además indicaron que los profesores de educación física les negaron su participación de acuerdo con su identidad sexual y una alta preocupación por su intimidad corporal en los camarines (Devis et al., 2018). González (2020) concluye que de acuerdo a los cambios que se han realizado por el Comité Olímpico Internacional a los sistemas de clasificación y categorización en evaluaciones de género trans a nivel deportivo competitivo, se espera una mayor aceptación de esta realidad, donde la Educación Física inicial reconozca y trabaje sobre el respeto y naturalidad de las diferencias biológicas, anatómicas, fisiológicas, psicológicas u otras y así no condicionar o predisponer la práctica de una actividad físico-deportiva a la heteronorma.

Con la finalidad de profundizar en el tema de la homofobia y transfobia en Educación Física es que surgen los objetivos de la presente investigación: i) validar dos escalas para indagar en las actitudes negativas hacia la homosexualidad y personas trans por parte de estudiantes de la carrera de pedagogía en Educación Física de Chile; ii) describir estas actitudes en una muestra de Santiago de Chile.

\section{Método}

\section{Muestra}

De tipo no probabilística intencionada (Maureira \& Flores, 2018). Estuvo constituida por 255 estudiantes de Pedagogía en Educación Física de tres universidades de Santiago de Chile. La edad mínima fue de 18 y la máxima de 32 años, con una media de 21.7 \pm 2.9 . Del total, 96 fueron hombres (37.6\%) y 159 fueron mujeres (62.4\%). 45 estudiantes cursaban primer año de la carrera (17.6\%), 39 segundo año (15.3\%), 73 tercer año (28.6\%), 53 cuarto año (20.8\%) y 45 quinto año (17.6\%).

\section{Instrumentos}

Se utilizó la escala de homofobia EHF-10 (Moral \& Ortega, 2008) que consta de 10 ítems. El contenido del instrumento incluye la aceptación/exclusión de personas homosexuales, de la homosexualidad como orientación sexual y las manifestaciones de la homosexualidad en público. La escala se contesta con un formato Likert de 1 (totalmente en desacuerdo) hasta 5 (totalmente de acuerdo). También se aplicó la escala de actitudes negativas hacia personas trans (EANT) (Páez et al., 2015) constituida por 9 ítems. El contenido del instrumento incluye actitudes hacia personas trans y transfobia. La escala se contesta con un formato tipo Likert de 1 (muy en desacuerdo) hasta 5 (muy de acuerdo).

\section{Procedimiento}

La escala de homofobia EHF-10 fue modificada redactando todos los ítems en negativo, de manera que puntajes más cercanos al 5 indica mayor grado de homofobia. En el ítem 6 la palabra ligarme fue reemplazada por seducirme en base al juicio de expertos. La escala de actitudes negativas hacia personas trans mantuvo los mismos ítems que el original.

Las dos escalas fueron aplicadas en forma online a una muestra piloto de 50 estudiantes de Pedagogía en Educación Física, los cuales no reportaron dudas sobre el lenguaje utilizado ni en la redacción de los ítems. Se realizaron los análisis preliminares de validez y confiabilidad del instrumento. Luego se procedió a aplicar el cuestionario al total de la muestra. Los índices de validez y confiabilidad final del instrumento se presentan en el apartado de resultados. Durante el presente estudio se respetaron los principios éticos para las investigaciones médicas con seres humanos de la Declaración de Helsinki (World Medical Asociation, 2013). Para participar en la presente investigación todos los estudiantes firmaron un consentimiento informado.

\section{Análisis de datos}

Para determinar los índices de validez de ambos instrumentos se aplicaron análisis factoriales exploratorios a través de un análisis de componentes principales con rotación ortogonal de Varimax. Para ello se utilizó el programa estadístico IBM SPSS versión 25.0 para Windows. Luego se realizaron análisis factoriales confirmatorios, donde se empleó el método de estimación de máxima verosimilitud (ML), los índices absolutos de chi cuadrado $\left(\mathrm{x}^{2}\right)$, el RMSEA (media de residuos estandarizados) y el CFI (ajuste comparado). Para ello se utilizó en programa estadístico SPSS AMOS 26.0 Los índices de confiabilidad se obtuvieron con la prueba Alfa de Cronbach.

Para la descripción de las puntuaciones de cada ítem se utilizaron porcentajes y se utilizó pruebas de $\mathrm{x}^{2}$ para comparar los puntajes obtenidos entre hombres y mujeres. Se consideró significativo valores $\mathrm{p}<.05$. 


\section{Resultados}

Propiedades psicométricas de la escala de homofobia EHF-10

La prueba de Kaiser-Mayer-Olkin (KMO) para el cuestionario muestra un valor de .803 y la prueba de esfericidad de Bartlett un $p=.000$ por lo que se procedió a realizar un análisis factorial exploratorio. Este entregó la existencia de tres factores que explican el $58,110 \%$ de la varianza total (tabla 1), con seis ítems que saturan en el factor 1 (orientación y homosexualidad en público), dos en el factor 2 (exclusión de personas homosexual) y tres en el factor 3 (ideas homosexuales). El alfa de Cronbach entrega un valor de .761 para los 10 ítems de la escala.

\begin{tabular}{|c|c|c|}
\hline & Factor 1 & Factor 2 Factor 3 \\
\hline Item 4. Respeto a los homosexuales y lesbianas siempre y cuando no se exhiban & .594 & \\
\hline Ítem 5. Una cosa es la homosexualidad, pero vestirse de mujer o cambiarse de sexo está en contra de la naturaleza & .651 & \\
\hline Ítem 6. Agrediría físicamente a una persona del mismo sexo si intentará "seducirme" & .646 & \\
\hline Ítem 8. No puedo ver a una pareja del mismo sexo besándose & .801 & \\
\hline Ítem 9. Si un amigo o amiga me dijera que es homosexual/lesbiana no podría darle mi apoyo & .687 & \\
\hline Ítem 10. Las parejas homosexuales/lesbianas no tienen derecho a adoptar o criar hijos o hijas & .694 & \\
\hline Ítem 2. Los homosexuales y lesbianas no deberían convivir con todas las personas & & .852 \\
\hline Ítem 3. Me avergonzaría tener un hermano homosexual o hermana lesbiana & & .734 \\
\hline Ítem 1. La sola idea de sentirme atraído/a físicamente por personas de mi propio sexo me pone nervioso/a & & .811 \\
\hline Ítem 7. No podría reconocer si he tenido sueños o fantasías eróticas con personas del mismo sexo & & .743 \\
\hline
\end{tabular}

En la figura 1 se presenta el modelo final de dimensiones e ítems para el cuestionario a partir de los tres factores extraídos del análisis factorial confirmatorio. Los resultados mostraron un $x^{2}$ significativo $\left(x^{2}=86.542\right.$; $p=.000)$, razón por la cual se observan otros valores para conocer el ajuste del modelo propuesto. Los valores del $\mathrm{RMSEA}=.080 \mathrm{y}$ del $\mathrm{CFI}=.905$ indican un ajuste adecuado del modelo (Maureira, 2016, Morata et al., 2015, Zubillaga \& Cañadas, 2021).

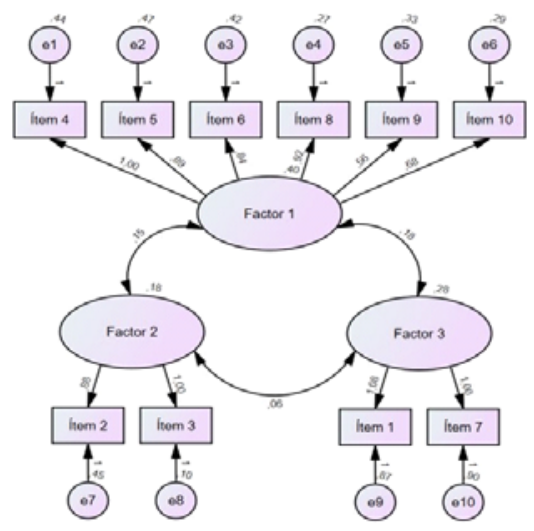

Figura 1. Análisis factorial confirmatorio de la escala de homofobia EHF-10.

Propiedades psicométricas de la escala de actitudes negativas hacia personas trans (EANT)

La prueba de Kaiser-Mayer-Olkin (KMO) para el cuestionario muestra un valor de .881 y la prueba de esfericidad de Bartlett un $p=.000$ por lo que se proce- dió a realizar un análisis factorial exploratorio. Este entregó la existencia de un factor que explican el 59.006\% de la varianza total (tabla 2). El alfa de Cronbach entrega un valor de 901 para los 9 ítems del cuestionario.

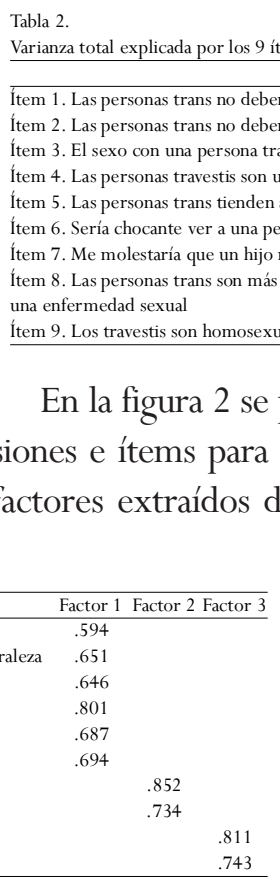
\& Cañadas, 2021).

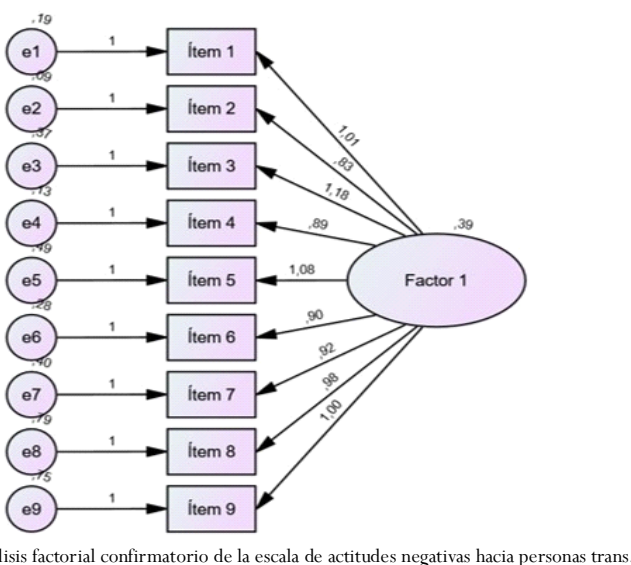

Figura 2. Análisis factorial confirmatorio de la escala de actitudes negativas hacia personas trans.

\section{Actitudes hacia la homosexualidad $y$ transexualidad}

En la tabla 3 se observan los porcentajes de respuestas a cada ítem de la escala de homofobia EHF-10. Es posible notar que el ítem 3. Me avergonzaría tener un hermano homosexual o hermana lesbiana posee el mayor porcentaje de respuestas totalmente en desacuerdo y en desacuerdo con el 96.9\%, seguida del ítem 2. Los homosexuales y lesbianas no deberían convivir con todas las personas con un $94.6 \%$ de respuestas totalmente en desacuerdo y en desacuerdo. Por el contrario, el ítem 1. La 
sola idea de sentirme atraído / a físicamente por personas de mi propio sexo me pone nervioso / a posee el mayor porcentaje de respuestas de acuerdo y totalmente de acuerdo con un $11.8 \%$, seguida del ítem 7. No podría reconocer si he tenido sueños o fantasías eróticas con personas del mismo sexo con un $8.2 \%$ de respuestas de acuerdo y totalmente de acuerdo. El ítem 1. La sola idea de sentirme atraído / a físicamente por personas de mi propio sexo me pone nervioso / a posee el mayor porcentaje de respuestas ni en desacuerdo ni de acuerdo con un 33.3\%, seguido del ítem 7. No podría reconocer si he tenido sueños o fantasías eróticas con personas del mismo sexo con un $21.2 \%$ de respuestas ni en desacuerdo ni de acuerdo.

Al comparar las respuestas entre hombres y mujeres no se observan diferencias en ninguna de los ítems de la escala de homofobia EHF-10.

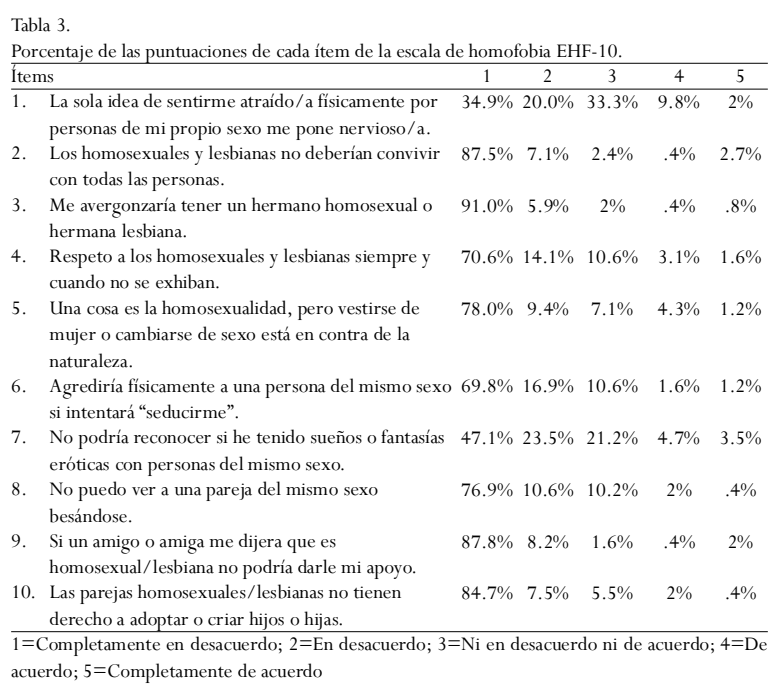

En la tabla 4 se observan los porcentajes de respuestas a cada ítem de la escala de actitudes negativas hacia personas trans. Es posible notar que el ítem 2. Las personas trans no deberían poder enseñar en las escuelas posee el mayor porcentaje de respuestas totalmente en desacuerdo y en desacuerdo con el 93.7\%, seguida del ítem 4. Las personas travestis son una amenaza para la familia y la sociedad con un $92.6 \%$ de respuestas totalmente en desacuerdo y en desacuerdo. Por el contrario, el ítem 9. Los travestis son homosexuales disfrazados de mujer posee el mayor porcentaje de respuestas de acuerdo y totalmente de acuerdo con un 7.9\%, seguida del ítem 8. Las personas trans son más propensas que el resto de la sociedad a contraer una enfermedad sexual con un $7.5 \%$ de respuestas de acuerdo y totalmente de acuerdo. El mismo ítem 8 posee el mayor porcentaje de respuestas ni en desacuerdo ni de acuerdo con un 21.2\%, seguido del ítem 9. Los travestis son homosexuales disfrazados de mujer con un 19.2\% de respuestas ni en desacuerdo ni de acuerdo.
Al comparar las respuestas entre hombres y mujeres sólo se observan diferencias significativas en el ítem 8. Las personas trans son más propensas que el resto de la sociedad a contraer una enfermedad sexual donde las mujeres presentan mayor cantidad de respuestas de acuerdo y totalmente de acuerdo $(\mathrm{p}=.033)$.

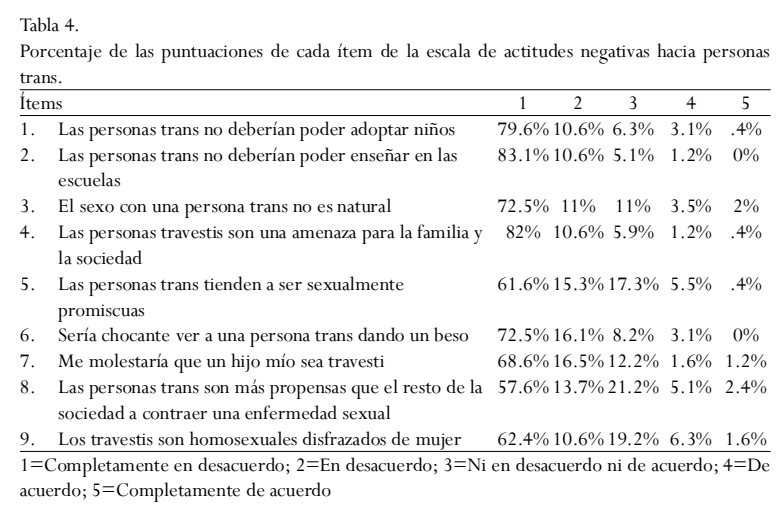

\section{Discusión}

Las escalas de homofobia EHF-10 y de actitudes negativas hacia personas trans han mostrado ser instrumentos válidos y confiables para recoger información sobre la orientación homosexual, manifestaciones homosexuales en público, exclusión de personas homosexuales y actitudes hacia personas transexuales. La primera escala presenta tres factores y la segunda un factor, en ambos casos se presenta una adecuada varianza explicada y una buena consistencia interna, lo cual muestra que son herramientas validas y confiables para evaluar a estudiantes de Educación Física. El presente estudio reveló una varianza total explicada del 58.1\% en la escala EHF-10 valor superior al 40.89\% del estudio original de Moral y Ortega (2008), pero una menor confiabilidad (.761 v/s .870). Misma situación observada con el trabajo de Moral y Martínez (2011) donde la escala EHF-10 presenta un 46.75\% de varianza explicada y un alfa de Cronbach de .870 . En relación con la escala de actitudes negativas hacia personas trans (Páez et al., 2015), esta investigación revelo valores de 59\% de varianza total explicada y .901 de alfa de Cronbach, mayores al $52.57 \%$ y .882 de la escala original. Otro estudio de Cáceres (2021) reveló valores adecuados de confiabilidad, la existencia de un solo factor y un valor de confiabilidad de .887 del EANT.

A diferencia de lo revelado en la bibliografía, el presente estudio mostró bajos niveles de homofobia y transfobia en la muestra. Sin embargo, algunos trabajos revelan que es posible expresar aceptación y normalización de estudiantes homosexuales, pero aún ejercer 
conductas discriminatorias (Márquez, 2017, Piedra et al.,2014). Esto podría explicar los bajos porcentajes de respuestas de aceptación de actitudes negativas hacia personas con diferentes orientaciones sexuales.

Otras investigaciones previas (Guevara, 2020; Silva, 2018; Márquez, 2017) coinciden mostrando rasgos de aceptación social a las personas homosexuales, pero rechazo o discriminación cuando los sujetos se perciben involucrados desde algún punto de vista, situación similar encontrada en este estudio. También se destaca que los prejuicios de las estudiantes mujeres hacia personas trans podrían ser discriminatorios. En términos generales, la tendencia de superioridad machista busca discriminar a las personas homosexuales y transexuales en nuestra sociedad. En estudiantes de Educación Física y en los deportes se generan ambientes sociales de manera natural para que dichas situaciones lamentablemente sucedan y es interesante conocer la conducta de hombres y mujeres al respecto.

Si bien es cierto, no se encontraron diferencias significativas entre hombres y mujeres, excepto en la idea que las personas trans son más propensas a adquirir una enfermedad sexual en relación con las otras personas de la sociedad, donde las mujeres están de acuerdo o totalmente de acuerdo en relación con esta afirmación. Estos resultados favorables hacia las personas trans puede deberse a que los últimos años ha existido un movimiento social de derechos y demandas reconociéndolo como una problemática social para disminuir la discriminación y exclusión (Páez, et al., 2015). Por otra parte, se puede reconocer en el contexto universitario los esfuerzos que han realizado para incorporar seminarios, congresos, charlas, cursos e incluso asignaturas para poner en mesa la temática. En este sentido Gutiérrez (2018) afirma que la falta de reconocimiento de los derechos de las personas trans se debe a la ausencia de políticas públicas adecuadas.

Respecto a la afirmación Los travestis son homosexuales disfrazados de mujer un estudio reveló que los vecinos de travestis los consideran agentes negativos en los espacios comunes (Rigueiral \& Seidmann, 2016), con ideas erróneas de quienes visten de una manera distinta a la de su sexo, concluyendo que el aspecto estético es decidor en la concepción del comportamiento de los individuos. Sobre lo mismo la simbolización cultural incluso publicitaria acrecienta el estereotipo sobre la imagen (Rodríguez, 2007), todas situaciones que pueden influenciar en el porcentaje de respuestas afirmativas encontradas en esta muestra.

\section{Conclusión}

Las escalas de homofobia EHF-10 y de actitudes negativas hacia personas trans poseen índices adecuados de validez y confiabilidad en una muestra de estudiantes de Educación Física. Su aplicación revela bajos niveles de homofobia y transfobia con menos de un 10\% de respuestas totalmente de acuerdo o de acuerdo frente a los ítems de actitudes negativas. Por el contrario, el rechazo a estas actitudes supera el 50\%, llegando al 90\% en algunos ítems. En la mayoría de las afirmaciones no se observaron diferencias entre hombres y mujeres.

Son necesarias investigaciones en muestras de mayor tamaño y de diferentes universidades, para tener una mirada más amplia de las actitudes negativas hacia la homosexualidad y personas trans por parte de estudiantes de Educación Física en todo Chile. Finalmente, se hace necesario observar a estos/as estudiantes en diferentes situaciones con personas con diversas orientaciones sexuales e identidades de género, para confirmar si su autopercepción de actitudes hacia la homofobia y transfobia se correlaciona con sus actitudes en situaciones reales.

\section{Referencias}

American PsychologicalAssociation(2011). Definition of terms:Sex, Gender, Gender Identity, Sexual Orientation. Disponible en: http: / / www.apa.org/pi/lgbt/resources/sexualitydefinitions.pdf

Ayvazo,S. \& Sutherland,S. (2009). Uncovering the secrets: Homophobia inPhysical Education. ActioninTeacher Education, 31(3), 56-69.https:/ /doi.org/10.1080/01626620.2009.10463528

Barber,H. \& Krane,V.(2007). Creating Inclusive and Positive Climates in Girls' andWomen's Sport: Position Statementon Homophobia, Homonegativism, and Heterosexism. Women in Sport \& Physical Activity Journal, 16(1), 53-56. https://doi.org/10.1123/ wspaj.16.1.53

Barbero, J. (2003). La educación física y el deporte como dispositivos normalizadores de la heterosexualidad, en O. Guaschy O.Viñuales (Eds.) Sexualidades:diversidad y control social (pp.355-377). Bellaterra.

Barrientos, J. \& Cárdenas, M.(2013). Homofobia y calidad de vida de gayy lesbianas: unamiradapsicosocial. Psykhe, 22(1), 3-14. http:// dx.doi.org/10.7764/psykhe.22.1.553

Barrientos, J., Espinoza-Tapia, R., Meza, P., Saiz, J., Cárdenas, M., Guzmán-González, M., Gómez, F., Bahamondes, J. \& Lovera, L. (2019). Efectos del prejuicio sexual en la salud mental depersonas transgénero chilenas desdeel modelo de estrés de las minorías: una aproximación cualitativa. TerapiaPsicológica,37(3), 181-197.

Beserra, J., Brito, A. \& Ribeiro, S. (2019). Homofobia nas aulas de educaçãofísica: um desafio para os professores de educação física do município de Buriti dos montes - Piaui. Revista do Plano Nacional de Formação de Professores da Educação Básica, 7(2), 81-90. 
Borrillo,D. (2010). Homofobia. Históriae crítica de um preconceito. Autêntica. Cáceres,A. (2021). Adaptación y validación de la Escala de Actitudes Negativas Hacia PersonasTrans (EANT) en jóvenes y adultos peruanos.Tesis de pregrado, Universidad CésarVallejos, Perú.

Camacho-Miñano, M. \& Girela-Rejón, M. (2017). Evaluación de una propuesta formativa sobre género en educación física para estudiantes de Ciencias deActividad Física y el Deporte. Cultura,Ciencia y Deporte, 12(36), 195-203. https://doi.org/10.12800/ cod.v12i36.950

Campo,A., Herazo, E. \& Oviedo, H. (2013). Sustantivos para definir homofobia. Revista Ciencias de la Salud, 11(3), 297-294.

Carmona, K. (2021). La violencia contra lasmujeres trans enlosespacios públicos: entre el acoso sexual y la transfobia. Revista LatinoamericanadeDerechosHumanos, 32(1), 1-43.https://dx.doi.org/10.15359/ rldh.32-1.1

Carvajal,Á.(2018).Transexualidad y transfobia en el sistema educativo. Revista Humanidades de la Universidad de Costa Rica, 8(1), 152-181. http://dx.doi.org/10.15517/h.v8i1.31467

Devís, J., Pereira, S., López, E., Pérez,V.\& Fuentes, J. (2018).Looking backintotranspersons' experiencesinheteronormative secondary physical education contexts. Physical Education and Sports Pedagogy, 23(1), 103-116. https://doi.org/10.1080/ 17408989.2017.1341477

García, P. (2005).Identidad de género:modelos explicativos. Escritos de Psicología, 7, 71-81.

Gill,D.,Morrow,R., Collins,K.,Lucey,A.\& Schultz,A.(2010).Perceived climate in physical activity settings. Journal of Homosexuality, 57(7), 895-913.https://doi.org/10.1080/00918369.2010.493431

González,J.(2020). Educación física y deporte trans-formado. Praxis, Educación y Pedagogía, 4, 78-97. https://doi.org/10.25100/ praxis_educacion.v0i4.9121

Guevara, R. (2020). Los roles de género y las actitudes frente a la homosexualidad masculina en estudiantes universitarios heterosexuales del primer y séptimo semestre de la carrera de Pedagogía de la Actividad Física y Deportes, de la Facultad de Ciencias Humanas y de la Educación, de la UniversidadTécnica de Ambato, CantónAmbato,provincia deTungurahua. Seminario para optar al título de Psicólogo Educativo y Orientador Vocacional,UniversidadTécnica deAmbato,Ecuador.

Gutiérrez,P.(2018).Aceptación, reconocimientoyaccionesafirmativas para las personas trans en la Uacj. Revista del Centro de Investigación y Docencia, 60, 53-72.

Lleixá,T., Soler, S. \& Serra, P. (2020). Perspectiva de género en la formación de maestras y maestros de educación física. Retos, 37, 634-642.https://doi.org/10.47197/retos.v37i37.74253

Márquez, J. (2017). Actitudes de los/as estudiantes de magisterio de la Universidad de Huelva hacia la homosexualidad. Ponencia presentada en el XIV Congreso Internacional deEducación Inclusiva: Prácticas innovadorasinclusivas: retosy oportunidades. Oviedo, España.

Maureira, F. (2016). Estadística avanzada para educación fisica. Editorial AcadémicaEspañola.

Maureira, F. (2020). Amor, sexo y cerebro. Bubok Publishing.

Maureira, F. \& Flores, E. (2018). Manual de investigación cuantitativa. BubokPublishing.

McCaughtry, N., Dillon,S.R., Jones, E.\& Smigell, S. (2005). Sexuality sensitiveschooling. Quest,57(4),426-443.https://doi.org/10.1080/ 00336297.2005 .10491865
Moral, J. \& Martínez, J. (2011). Escala de actitud hacia la homosexualidad: propiedades psicométricas y aspectos diferenciales por sexos. SOCIOTAM,21(1), 105-124.

Moral, J. \& Ortega, M. (2008). Diferencias de género en representación social delasexualidad, asícomo enactitudes yconductas sexuales. Estudios sobre las Culturas Contemporáneas, 14(28), 97-119.

Morata, M., Holgado, F., Barbero, I. \& Méndez, G. (2015). Análisis factorial confirmatorio. recomendaciones sobre mínimos cuadrados no ponderados en función del error tipo I de ji-cuadrado y RMSEA. Acción Psicológica, 12(1), 79-90. https:/ / dx.doi.org/ 10.5944/ap.12.1.14362

O’Brien, K., Shovelton, H. \& Latner, J.(2013). Homophobiain physical education and sport:The role of physical/sporting identity and attributes, authoritarianaggression, and social dominance orientation. International Journal of Psychology, 48(5), 891-899. https: / / doi.org/ 10.1080/00207594.2012.713107

Páez,J.,Hevia, G., Pesci, F. \& Rabbia,H. (2015). Construcción y validación de una escala deactitudesnegativashaciapersonas trans. Revista dePsicología(PUCP), 33(1), 153-190.

Pérez,V.,Fuentes,J., Pereira, S.\& Devís,J. (2016).Abjectionandalterity in the imagining of transgender in physical education and sport: a pedagogical approachinhighereducation. Sport, Education and Society, 21(7), 985-1002. https://doi.org/10.1080/ 13573322.2014.981253

Piedra, J., Ramírez, G., Ries,F., Rodríguez,A. \& Phipps C. (2016). Homophobiaandheterosexism: Spanishphysical education teachers' perceptions. Sport in Society, 19(8-9), 1156-1170.https://doi.org/ 10.1080/17430437.2015.1096257

Piedra,J., Rodríguez,A. \& Latorre,A. (2014).Visibilizando lo invisible: creencias del profesorado de educación física sobre homofobia y masculinidad. Retos, 25, 44-49. https://doi.org/10.47197/ retos.v0i25.34472

Piedra, J., Rodríguez,A., Ries, F. \& Ramírez, G. (2013). Homofobia, heterosexismo yeducaciónfísica:percepciones del alumnado.Profesorado, 17(1), 325-338.

Rigueiral, G. \& Seidmann, S. (2016) Análisis crítico de los principales estudios sobre las personas trans. Disponible en https:// www.aacademica.org/000-044/575.pdf

Rodríguez,T.(2007). Representaciones sociales:Teoría e investigación. Universidad de Guadalajara.

Serra, P., Prat, M., Nicolino,A., Soler, S. \& Silva,A. (2020). Género y curriculum de formación del profesorado en Educación Física: un diálogo entre Brasil y España. Revista Iberoamericana de Educación, 82(2), 191-210.https://doi.org/10.35362/rie8223637

Silva, P., Jaeger,A. \&Valdivia, P.(2018). Percepción de los estudiantes sobre comportamientos homofóbicos y heterosexistas en educación física. Revista de Psicología del Deporte, 27(2), 39-46.

WorldMedicalAsociation.(2013).WorldMedicalAssociationDeclaration of HelsinkiEthical Principlesfor Medical Research Involving Human Subjects. JAMA, 310(20), 2191-2194. https://doi.org/10.1001/ jama.2013.281053

Zubillaga, M. \& Cañadas, L. (2021). Diseñoy validación delcuestionario «\#EvalEF» para conocer el proceso de evaluación desarrolladopor losdocentes de educaciónfísica. Retos, 42, 47-55.https: / /doi.org/ 10.47197/retos.v42i0.86627 